\title{
Reduction of Stress of Seated Reading Postures for Desk with Built-in Bookholder and Desktop-mounted Sliding Drawer
}

\author{
Dohyung Kee, Bo Gyung Jeong, Jae Chun Lee, Kyung Min Hwang, Han Byel Lee
}

Department of Industrial and Management Engineering, Keimyung University, Daegu, 704-701

\author{
Corresponding Author \\ Dohyung Kee \\ Department of Industrial and \\ Management Engineering, Keimyung \\ University, Daegu, 704-701 \\ Mobile : +82-10-5656-1260 \\ Email : dhkee@kmu.ac.kr
}

Received : February 13, 2014

Revised : April 05, 2014

Accepted : April 08, 2014
Objective: This study aims to develop a new desk with a built-in bookholder and desktop-mounted sliding drawer for reducing stress of seated reading postures and to analyze its effect.

Background: It is general for readers to bend their back and neck when reading books on desks, which may result in high back and neck postural stress. In addition, postures using vertically layered drawers positioned at the side of users may be awkward.

Method: For reducing the postural stress, a new desk with a built-in bookholder and sliding drawer was developed based on a standard of KS G 4208 for student desk. The muscle activities of reading postures on both existing and new desks were measured using EMG, in which seven male and three female college students participated. The postural stress, and musculoskeletal discomfort and ease of usage for existing and new drawers were analyzed by RULA and questionnaire survey, respectively.

Results: Compared to existing desk, the muscle activity for the new desk with a bookholder was reduced by $47 \%$ when reading books. In addition, musculoskeletal discomforts in the shoulder, neck and low back were significantly lower when using the new desk with a bookholder. Although the stresses for postures using the desktopmounted sliding drawer and general drawer were not significantly different, the questionnaire survey revealed that the new desktop-mounted sliding drawer is easier to use.

Conclusion: A new desk developed in this study significantly reduced postural stress and enhanced subjective preference when reading.

Application: This would be useful when developing new desks with low postural stress.

Keywords: Desk, Bookholder, Sliding drawer, Reading posture, EMG

\section{Introduction}

The muscular strength, especially, neck and low back muscular strengths of the students leading a life sitting in front of a desk most of the day are greatly weak. In general, students slightly bend at lower back, and thus, shoulders lean forward, and neck moves forward like a turtle's neck, or hips are barely positioned in the chair. In 
this way, they take a posture adding burden to the lower back and neck (Chosun Ilbo, 2013a).

Korea's herniated cervical disc patients show a rapidly increasing trend in view of a $37 \%$ surge from some 570,000 patients in 2007 to some 780,000 in 2011 (Chosun Ilbo, 2013b). Neck pain including the herniated cervical disc is the disease that about 67\% of total population experiences once or more in life, and if the disease becomes chronicle one, serious problems can be caused to the quality of life (Bovim, Schrader and Sand, 1994; Cote, Cassidy and Carroll, 1998; Yoo, 2008; Wang et al., 2003). Continuous flexion behavior of cervical spine increases load to neck tissues (Gooch 1993; Harms-Ringdahl and Ekholm 1986; Twomey and Taylor 1982), and this phenomenon increases activity of neck bone muscles (Schuldt et al., 1986). To check all these, Yoon (2002) measured muscle activity around neck including splenius capitis, strenocleido mastoideus, trapezius, and deltoid. Park (2005) examined muscle activity of neck and shoulders in the forward leaning head posture. Aspden (1992) asserted that pain can be caused, due to excessive tension of trapezius and surrounding muscles, when using a cellphone or reading a book on the desk. However, an effort to reduce huge load to the reading posture on such a desk cannot be found easily.

This research aims to develop a desk with a built-in bookholder and a desktop mounted sliding drawer that can reduce loads to the neck and shoulders in a student's book reading posture on the desk at school and at home for a long time, and to analyze the effects in a quantitative/qualitative manner.

\section{Methods}

\subsection{Development of new desk}

To improve studying or book-reading posture using a desk, this research developed a new desk for home learning with a built-in bookholder and a desktop mounted sliding drawer on the top surface of the desk. The dimensions of the new desk were decided by referring to standard for student desks for domestic use (KS G 4208) (KATS, 2010).

\subsection{Validation experiment}

\subsubsection{Measurement of muscle activity}

The neck muscle activity before and after using a bookholder was measured using EMG (BIOPAC System Inc., CA, USA). This research selected splenius capitis, which is known that cumulative pain is generated much among several neck muscles (Chaffin and Andersson, 1991), and Figure 1 shows the surface electrode attachment locations and measuring postures for muscle activity. Ten healthy collegians ( 7 males and 3 females), who had never experienced musculoskeletal disorders at necks and shoulders for the past 1 year, participated in the muscle activity measurement. The measurement of muscle activity before and after using the built-in bookholder was conducted for two days. The sequence was randomly presented, according to the subjects. EMG signals were measured for 30 minutes, and the average value of amplitude was used as a dependent variable.

\subsubsection{Questionnaire survey}

After letting subjects read a book with his/her own posture by not using and using the bookholder for 30 minutes, respectively, this research conducted a questionnaire survey composed of 6 questions. The questionnaire consisted of the questions asking about shoulder wrick, stiff neck, stiff lower back, eye fatigue, the degree of concentration, and easiness of drawer use. The questions were composed of a 5-point Likert scale (5: very so, 4: yes, 3: fair, 2: no, 1: not very so). Thirty collegians (15 males, and 15 females) participated in the questionnaire survey. 


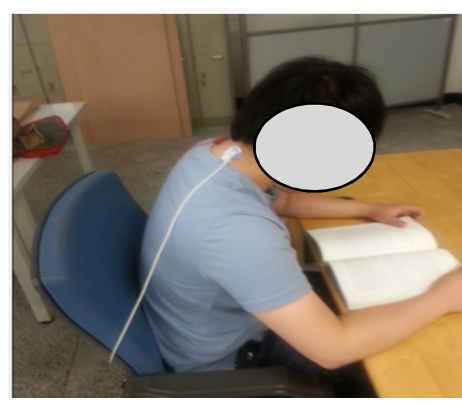

(a) Reading on desk

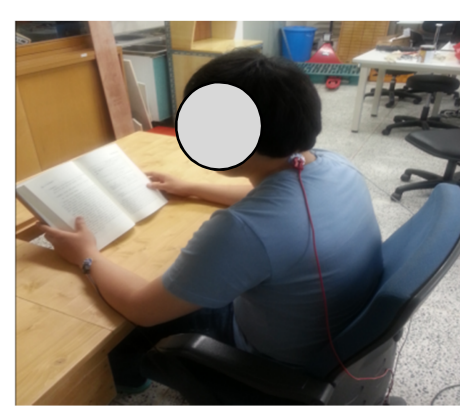

(b) Reading on bookholder

Figure 1. Scene of EMG experiment

\subsubsection{Desktop-Mounted sliding drawer}

The effect of a drawer developed in this research was evaluated by measuring the load of posture pulling out a book from the existing type of drawer installed vertically from the desk, and the sliding drawer mounted on the desk surface developed in this research. The posture load was measured using RULA (McAtamney and Corlett, 1993), which is known to be suitable for load evaluation of sitting posture like VDT work.

\section{Results}

\subsection{New desk}

The dimensions of the desk with a built-in bookholder on the desk surface are shown in Table 1 . The desk dimensions have been based on the standard for student desks for domestic use (KS G 4208). Instead of the existing drawer to pull out something by opening the drawer bending one's trunk to the side, the desk developed in this research has a mounted sliding door on the surface of the desk so as to improve a posture to pull out something from the drawer. The desk was manufactured by commissioning to a professional manufacturer (Figure 2), and the cost was about KRW 700,000.

\subsection{Effects of bookholder}

\subsubsection{EMG amplitude}

Figure 3 shows the amplitude trend and size of subject 1's splenius capitis before and after using the bookholder on reading posture. Compared to the state before using the bookholder, the amplitude of subject 1's splenius capitis became smaller. Ten subjects' average amplitude fell about $47 \%$ in the case of using a bookholder, compared to the state before using the bookholder

Table 1. Desk dimensions

\begin{tabular}{l|c}
\hline & Dimension (mm) \\
\hline Desk & $1350 \times 700 \times 750$ (width*depth*height) \\
\hline Bookholder & $300 \times 200$ (width*depth) \\
\hline Drawer & $250 \times 350 \times 250$ (width*depth*height) \\
\hline
\end{tabular}




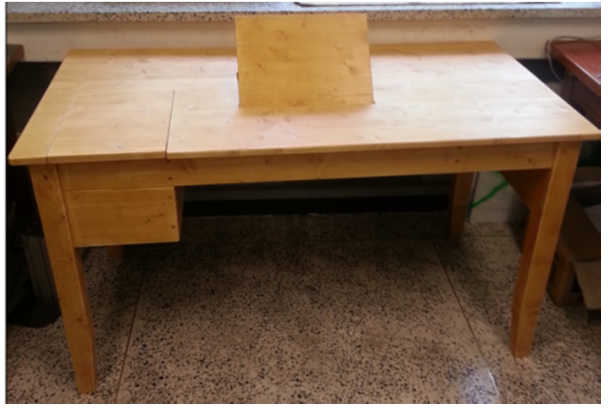

(a) No-bookholder

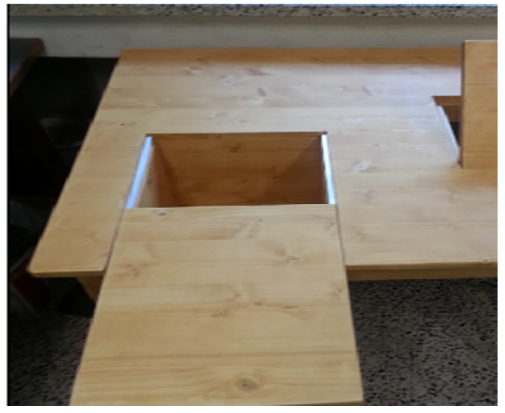

(b) Bookholder

Figure 2. New desk with bookholder and sliding drawer

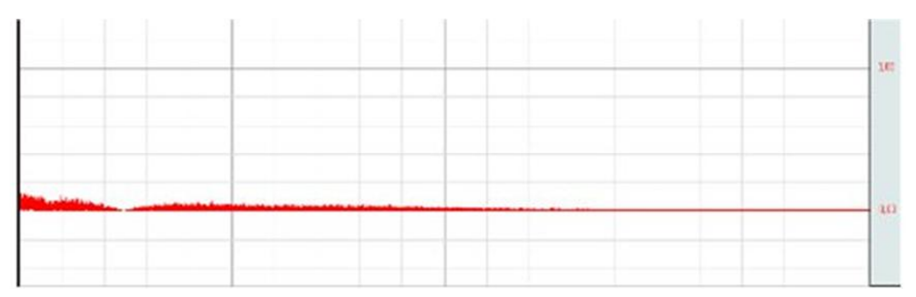

(a) No-bookholder

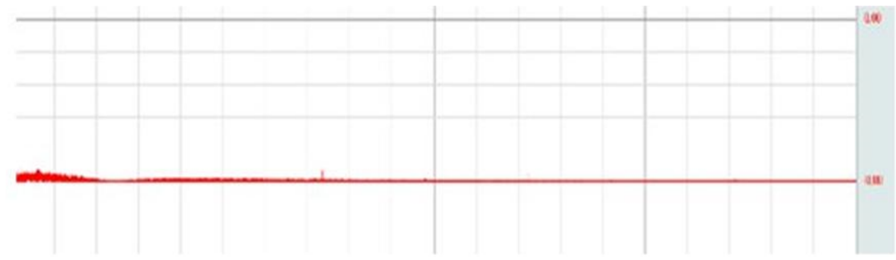

(b) Bookholder

Figure 3. EMG amplitude for subject 1

Table 2. EMG amplitude

\begin{tabular}{l|c|c|l|c|c}
\hline \multirow{2}{*}{} & \multicolumn{2}{|c|}{ Average amplitude (mv) } & \multicolumn{2}{c}{ Average amplitude (mv) } \\
\cline { 2 - 3 } \cline { 5 - 6 } & No bookholder & Bookholder & & No bookholder & Bookholder \\
\hline Subject1 & 0.00122 & 0.00059 & Subject7 & 0.00151 & 0.00091 \\
\hline Subject2 & 0.00131 & 0.00062 & Subject8 & 0.00134 & 0.00066 \\
\hline Subject3 & 0.00161 & 0.00098 & Subject9 & 0.00121 & 0.0006 \\
\hline Subject4 & 0.0011 & 0.00049 & Subject10 & 0.00142 & 0.00089 \\
\hline Subject5 & 0.00133 & 0.00062 & Average & 0.001349 & 0.000723 \\
\hline Subject6 & 0.00144 & 0.00087 & Std deviation & 0.000152 & 0.000171 \\
\hline
\end{tabular}


(Table 2, Figure 4). As a result of a pair-wise $t$-test, there was a significant difference of amplitude before and after using the bookholder $(p<0.01)$.

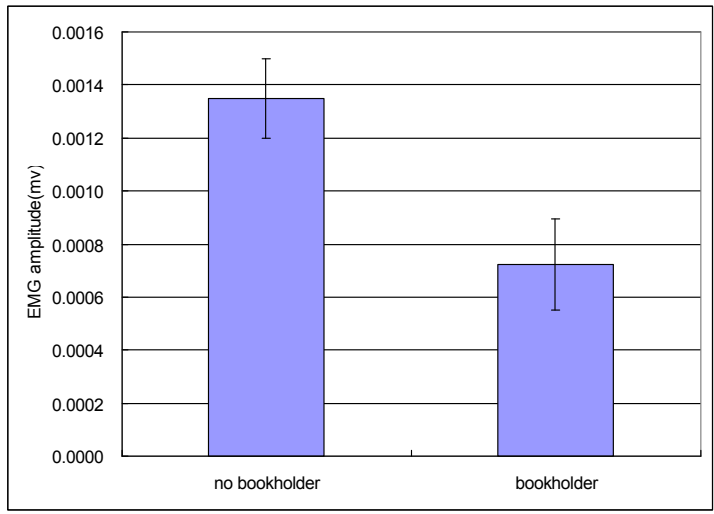

Figure 4. EMG activity for no-bookholder and bookholder

\subsubsection{Questionnaire survey}

Among the 5 questions related to the use of the bookholder, the subjects' responses before and after the use of the bookholder were different at significance level $1 \%$ as a result of the $t$-test concerning the three questions, namely, shoulder wrick $(p<0.01)$ stiff neck $(p<0.01)$, and stiff lower back $(p<0.01)$. Compared to the state before using the bookholder, when the subjects used the bookholder, subjective discomforts like shoulder wrick, stiff neck, and stiff lower back diminished. Meanwhile, eye fatigue $(p>0.76)$, and the degree of concentration $(p>0.30)$ did not show significance differences before and after using the bookholder, which implied they had no relevance with the use of a bookholder.

\subsection{Effects of desktop-mounted drawer}

The postures using the desktop-mounted sliding drawer developed in this research and the existing 2 or 3-layered sliding drawers attached vertically on the left or right side of a desk are exhibited in Figure 5. As a result of analyzing two postures using RULA,

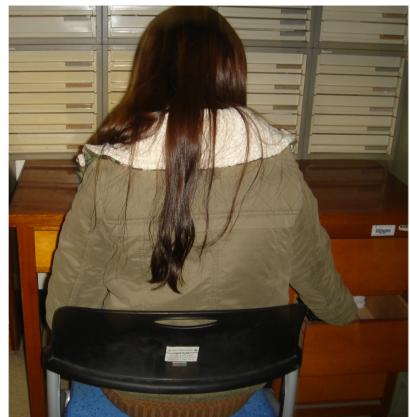

(a) Existing drawer

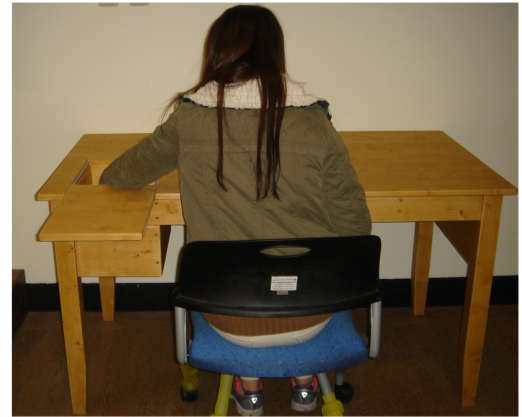

(b) New drawer

Figure 5. Postures using existing and new drawers 
two postures' action category was 2 (grand score: 3, respectively), and thus, no difference was shown.

In the questionnaire survey on the easiness of using two types of drawers, it was surveyed that the desktop-mounted sliding drawer developed in this research was felt easier in using than the existing drawer $(p<0.01)$ (Figure 6).

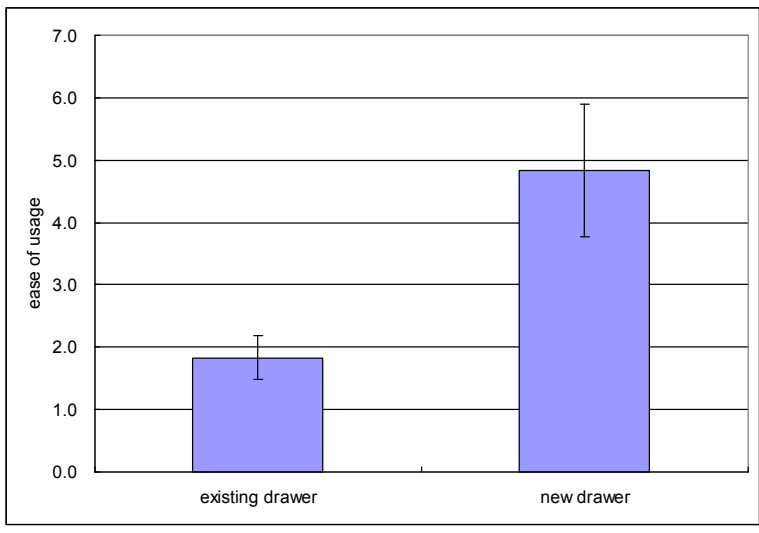

Figure 6. Ease of usage for drawers

\section{Conclusion}

This research developed a desk with a built-in bookholder on the desk surface and a desktop-mounted sliding drawer so as to reduce loads to neck and lower back, when one studies or reads a book using the desk for home learning, and evaluated the effects. The EMG amplitude of splenius capitis in a book reading posture dropped by about $47 \%$, when one used the bookholder developed in this research, compared to reading a book on a desk. The questionnaire survey revealed that shoulder wrick, stiff neck, and stiff lower back significantly improved statistically. In the postural load analysis using RULA for a posture pulling out something from a drawer, no difference was shown between this research-developed desktop-mounted sliding drawer and the existing desk drawer. However, the drawer developed in this research was felt easier in using, compared to the existing drawer in the questionnaire survey.

\section{References}

Aspden, R., Review of the functional anatomy of the spinal ligaments and the lumbar erector spinae muscles, Clinical Anatomy, 372-387, 1992.

Bovim, G., Schrader, H. and Sand. T., Neck pain in the general population, Spine, 19(12), 1307-1309, 1994.

Chaffin, D.B., Andersson, G.B.J. and Martin, B.J., Occupational Biomechanics, 3rd ed., Wiley \& Sons, 1999.

Chosun Ilbo Home Page, http://health.chosun.com/site/data/html_dir/2013/11/05/2013110501892.html (November 25, 2013a)

Chosun Ilbo Home Page, http://news.chosun.com/site/data/html_dir/2013/07/15/2013071500217.html (November 25, 2013b) 
Cote, P., Cassidy, J.D. and Carroll, L., The Saskatchewan health and back pain survey: The prevalence of neck pain and related disability in Saskatchewan adults, Spine, 23(15), 1689-1698, 1998.

Gooch, L., A Controlled Study of Sustained Cervical Spine Flexion, Master Thesis, Curtin University of Technology, Western Australia, 1993.

Harms-Ringdahl, K. and Ekholm, J., Intensity and character of pain and muscular activity levels elicited by maintained extreme flexion position of the power-cervical-upper-thoracic spine, Scandinavian Journal of Rehabilitation Medicine, 18(3), 117-126, 1986.

KATS (Korean Agency for technology and Standard), Student desks for domestic use (KS G 4208), 2010.

McAtamney, L. and Corlett, E.N., RULA: a survey method for the investigation of work-related upper limb disorders, Applied Ergonomics, 24(2), 91-99, 1993.

Park. S.D., Effects of neck postures on muscle activity of neck and shoulder in computer typing tasks, Master Thesis, Yonsei University, 2005.

Schuldt, K., Ekholm, J., Harms-Ringdahl, K., Nemeth, G. and Arborelius, U.P., Effects of changes in sitting work posture on static neck and shoulder muscle activity, Ergonomics, 29(12), 1525-1537, 1986.

Twomey, L. and Taylor, J., Flexion creep deformation and hysteresis in the lumbar vertebral column, Spine, 7, 116-122, 1982.

Yoo, C-U., Eletromyographic activity of the neck and shoulder muscles while watching a DMB phone with the neck flexed, Master Thesis, The Graduate School of Health and Environment, Yonsei University, 2008.

Yoon, D.K., A study on changes of workstress according to positions of monitor and mouse in computer tasks, Master Thesis, Incheon University, 2002.

Wang, W.T.J, Olson, S.L., Campbell, A.H., Hanten, W.P. and Gleeson, P.B., Effectiveness of physical therapy for patients with neck pain: An individualized approach using a clinical decision-making algorithm, American Journal of Physical Medicine \& Rehabilitation 82(3), 203-218, 2003.

\section{Author listings}

Dohyung Kee: dhkee@kmu.ac.kr

Highest degree: Ph.D., Department of Industrial Engineering, POSTECH

Position title: Professor, Department of Industrial and Management Engineering, Keimyung University

Areas of interest: Population stereotype, Product design, Posture classification scheme, Industrial safety, Musculoskeletal disorders

Bo Gyung Jeong: jbg355@kmu.ac.kr

Position title: College Student

Areas of interest: Ergonomics, Industrial Engineering 
Jae Chun Lee: ljc5217@gmail.com

Position title: College Student

Areas of interest: Ergonomics, Industrial Engineering

Kyung Min Hwang: hkm0117@kmu.ac.kr

Position title: College Student

Areas of interest: Ergonomics, Industrial Engineering

Han Byel Lee: teittl@nate.com

Position title: College Student

Areas of interest: Ergonomics, Industrial Engineering 